\title{
Analysis of inversion error characteristics of stellar occultation simulation data
}

\author{
MingChen Sun ${ }^{1,2}{ }^{*}$, QingLin Zhu' ${ }^{1}$ Xiang Dong ${ }^{1}$, and JiaJi Wu ${ }^{2}$ \\ 'China Research Institute of Radiowave Propagation, Qingdao 266107, China; \\ ${ }^{2}$ Xidian University, Xi'an 710126, China
}

\section{Key Points:}

- Stellar occultation simulation and measured data were inverted to verify the reliability of an onion-peeling algorithm.

- The effects of a priori data, the shape of the spectral absorption line, and the Earth's ellipticity on the accuracy of the inversion were analyzed.

Citation: Sun, M. C., Zhu, Q. L., Dong, X., and Wu, J. J. (2022). Analysis of inversion error characteristics of stellar occultation simulation data. Earth Planet. Phys., 6(1), 61-69. http://doi.org/10.26464/epp2022013

\begin{abstract}
Atmospheric stellar occultation observation technology is an advanced space-based detection technology that can measure the vertical distribution of trace gas composition, temperature, and aerosol content in a planet's atmosphere. In this study, an inversion algorithm of the onion-peeling method was constructed to invert the transmittance obtained from the forward mask. The method used a three-dimensional ray-tracing simulation to obtain the transmission path of the light in the Earth's atmosphere. The relevant parameters were then combined in the high-resolution transmission molecular absorption (HITRAN) database, and line-by-line integration was performed to calculate the atmospheric transmittance. The transmittance value was then used as an input to calculate the vertical distribution of oxygen molecules when using the single-wavelength inversion of the onion-peeling method. Finally, the oxygen molecule content was compared with the value attained by the Mass Spectrometer and Incoherent Scatter Radar Extended (MSISE00) atmospheric model to determine the relative error of our model. The maximum error was found to be $0.3 \%$, which is low enough to verify the reliability of our algorithm. Using Global-scale Observations of the Limb and Disk (GOLD) measured data to invert the oxygen number density, we calculated its relative deviation from the published result to further verify the algorithm. The inversion result was affected by factors such as prior data, the absorption spectral line type, the ellipticity of the Earth, and the accuracy of the orbit. Analysis of these error-influencing factors showed that the seasons and the Earth's ellipticity affected the accuracy of the model only $0.001 \%$ and could therefore be ignored. However, latitude and solar activity had a greater impact on accuracy, on the order of $0.1 \%$. The absorption line type affected the accuracy of the model by as much as $1 \%$. All three of these factors therefore need to be considered during the inversion process.
\end{abstract}

Keywords: stellar occultation; onion-peeling; GOLD; inversion; error

\section{Introduction}

Stellar occultation technology is an advanced, space-based detection technology. It uses the absorption of the spectrum of stars by planetary atmospheres to detect the vertical distribution of atmospheric parameters such as trace gas components, temperature, and aerosols (Roble and Hays, 1972). The advantages of this technology include self-calibration, global coverage, and a large amount of detection data (Kyrölä et al., 2004). It also plays an important role in research on the space environment (Bertaux et al., 2010) and space weather (Greer et al., 2018) of the Earth's middle and upper atmosphere.

Stellar occultation technology was first proposed by Hays and Roble (1968), and it was successfully used to detect the vertical

Correspondence to: M. C. Sun, 2297215868@qq.com

Received 27 SEP 2021; Accepted 10 NOV 2021.

Accepted article online 06 JAN 2022.

(C)2022 by Earth and Planetary Physics. distribution of ozone in the Earth's atmosphere (Ratier et al., 1999). Since then, stellar occultation technology has been used to study the atmospheres of Venus (Bertaux et al., 2007), Mars (Forget et al., 2009), Jupiter (Festou et al., 1981), and the Earth (Bertaux et al., 2004). A series of space environmental studies have been based on the detection of molecules and ions in the Earth's atmosphere, including those on atmospheric model improvement, trend prediction (Swartz et al., 2002), and the interaction between solar activity and planetary atmospheres.

Oxygen is one of the most important atmospheric components and is of significant scientific value (Ning LX et al., 2001). For example, the density profile of oxygen can be used to create panoramic images of atmospheric temperature to an altitude of $200 \mathrm{~km}$, and the distribution of oxygen can reflect atmospheric responses to geomagnetic interference and changes in solar ultraviolet levels (Eastes, 2009). Oxygen can also be used to study how atmospheric temperature and composition are affected by geomag- 
netic storms and how atmospheres respond to changes in the sun's extreme ultraviolet light on a global scale (Lumpe et al., 2007). As a result, the simulations in this study used one of the main absorption wavelengths of oxygen, $761.4 \mathrm{~nm}$, as the center wavelength.

In this study, we introduce an onion-peeling inversion algorithm that uses forward simulation transmittance to determine the vertical distribution of the molecular oxygen number density in the Earth's atmosphere. This method has the advantages of high inversion accuracy and easy implementation. To calculate the relative error in our algorithm, we compared our oxygen number density value with the value calculated by the Mass Spectrometer and Incoherent Scatter Radar Extended (MSISE00) atmospheric model. This allowed us to verify the reliability of our algorithm. The Global-scale Observations of the Limb and Disk (GOLD) stellar occultation observation data was used for the inversion, and the obtained oxygen density was compared with the released Level 2 data. The monthly average relative deviation was also calculated to further verify the accuracy of the algorithm.

Season, latitude, and the $F_{10.7}$ index (solar radio flux at $10.7 \mathrm{~cm}$ ) are factors that can affect the data used in the inversion calculation. To assess the dependency of the inversion error on these factors, the inversion error and distribution characteristics were calculated for different seasons, latitudes, and $F_{10.7}$ indexes. The influence of the absorption line type on the error was also analyzed because the spectral line-broadening mechanism changes with altitude; therefore, different altitudes correspond to different absorption line types. Finally, because of the ellipticity of the Earth and atmosphere, a local arc correction was performed to determine the effect of ellipticity on the inversion error.

\section{Inversion of the Simulated and Measured Stellar Occultation Data by the Onion-Peeling Method}

The equation for stellar occultation inversion (Kyrölä et al., 2010; Equation (1)) assumes that the conditions of a known atmospheric transmittance correspond to the tangent points of a specific atmospheric absorption component and the corresponding absorption cross section:

$$
T_{\text {abs }}=\exp \left(-\sum_{i} \sigma_{i} N_{i}(z)\right)=\exp \left(-\sum_{i} \int_{i} \sigma_{i}(\lambda, T(r(s))) \rho_{i}(r(s)) \mathrm{d} s\right),
$$

where $T_{\text {abs }}$ is transmittance, which is a function of molecular absorption; $\sigma_{i}$ is the absorption cross section of a specific absorption component; $\rho_{i}$ is the number density of the atmospheric absorption component and is related to the light transmission path; and $s$ is the light transmission path. The absorption cross section is related to the temperature and the absorption wavelength of the molecule and is used to measure the absorption capacity of a molecule.

The transmittance used in this study was based on simulation calculations, whereas the absorption cross section was calculated by line-by-line integration (Zhang $\mathrm{H}$ and Shi GY, 2000) and the relevant parameters in the high-resolution transmission molecular absorption (HITRAN) database (Qi RB et al., 2015). Temperature and molecular density data were a priori data and were taken from the MSISE00 atmospheric model. The specific transmission path of the light rays was simulated by using a three-dimensional ray-tracing method (Sun MC et al., 2020b), and a forward mask calculation was performed according to the principle shown above. The change in transmittance of the $761.4 \mathrm{~nm}$ wavelength of light and the absorption cross section of oxygen molecules with altitude are shown in Figures 1 and 2, respectively.

An onion-peeling method (Yee et al., 2002) for stellar occultation was constructed according to the input data. In this method, the Earth's atmosphere was layered, and local arcs were assumed to be symmetrical. The density of each layer was considered uniform, and the absorption cross-section of molecules above $200 \mathrm{~km}$ was considered to be zero. A schematic diagram for the method is shown in Figure 3, and the inversion calculation is shown in Equation (2):

$$
\begin{aligned}
\frac{1}{2} T_{i} & -\sigma_{1} \rho_{1}\left(S_{i}-\sqrt{R_{1}^{2}-R_{i}^{2}}\right)-\sum_{j=2}^{i} \sigma_{j} \rho_{j}\left(\sqrt{R_{j-1}^{2}-R_{i}^{2}}-\sqrt{R_{j}^{2}-R_{i}^{2}}\right) \\
& =\sigma_{i} \rho_{i} \sqrt{R_{i-1}^{2}-R_{i}^{2}}
\end{aligned}
$$

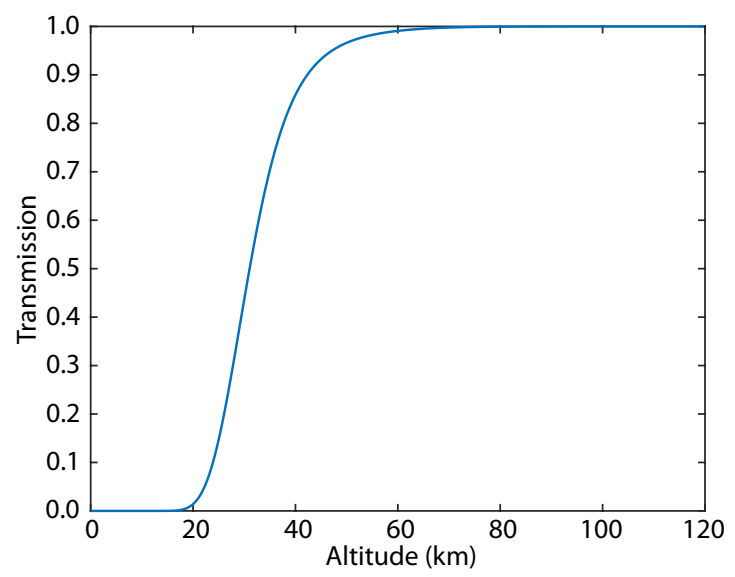

Figure 1. Variation in transmittance of $761.4 \mathrm{~nm}$ wavelength light with altitude. Transmission increases rapidly above $20 \mathrm{~km}$, and complete transmission is reached by approximately $60 \mathrm{~km}$.

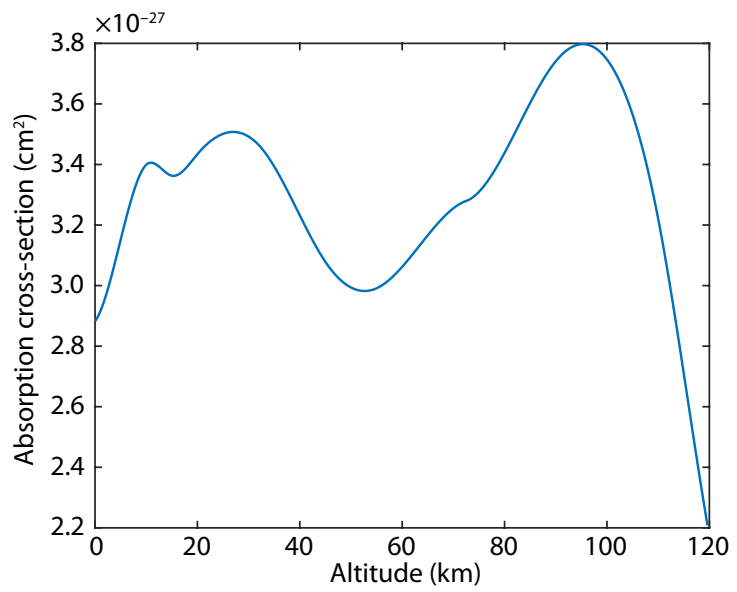

Figure 2. Variation in the absorption cross section of oxygen molecules with altitude. The absorption cross-section has two main peaks at approximately 25 and $95 \mathrm{~km}$ and two minor peaks at approximately 10 and $70 \mathrm{~km}$. 


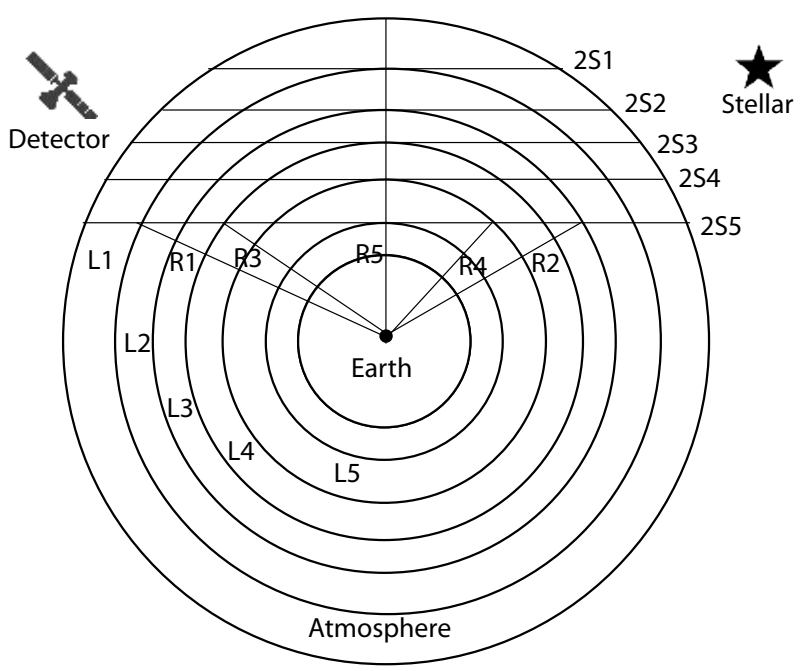

Figure 3. Schematic diagram of the onion-peeling method. Beginning at the outermost atmospheric layer (L1) and working down, the influence of the previous layer was subtracted to obtain the result for each layer (DeMajistre and Yee, 2002).

where $i \in(1, n), S$ is the length of the integral path along the ray direction, and $R$ is the height of the tangent point of the occultation event.

The oxygen molecular number density distribution was obtained by using the method described above, and it was compared with the value from the MSISE00 model to determine the error (Equation (3)):

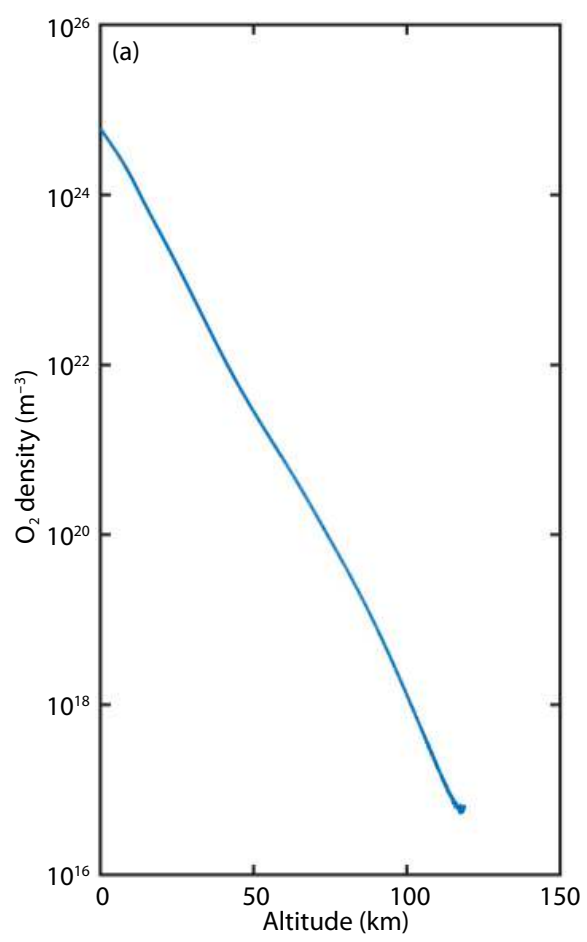

$$
R_{\text {relative }}=\frac{\rho_{\text {simulation }}-\rho_{\text {MSISE }}}{\rho_{\text {MSISE }}} \times 100 \%
$$

A positive or negative relative error indicated the degree to which our calculated oxygen molecular number density was greater than or less than the value from the MSISE00 model, respectively. Figure $4 \mathrm{~b}$ shows the change in the relative error with altitude.

Figure $4 \mathrm{~b}$ shows that the inversion error increases as the altitude increases. This effect is due to a number of factors, including the atmospheric refraction effect as well as the decrease in oxygen density with altitude (Qian LY et al., 2009; Figure 4a). These factors made it more difficult to determine oxygen content at higher altitudes, resulting in an increase in error. The inversion error also increases with altitude because of noise in the onion-peeling method. The largest observed inversion error was only $0.3 \%$, which shows that the inversion algorithm presented in this study is reliable.

To further verify the algorithm procedure, GOLD stellar occultation detection data (Eastes et al., 2017) were used to invert the oxygen number density, with an inversion wavelength of $142 \mathrm{~nm}$ and an altitude range of $150-200 \mathrm{~km}$. The monthly average deviation between the inversion result and the Level 2 data (Lumpe et al., 2016) was calculated, and the data for March, June, September, and December are shown in Figure 5. The data sample sizes for March, June, September, and December 2019 were 34, 34, 32, and 22 , respectively.

Analysis of the monthly average deviation showed that below 200 $\mathrm{km}$, the maximum deviation was $15 \%$. Only observation data at altitudes below $200 \mathrm{~km}$ were considered, based on the credibility

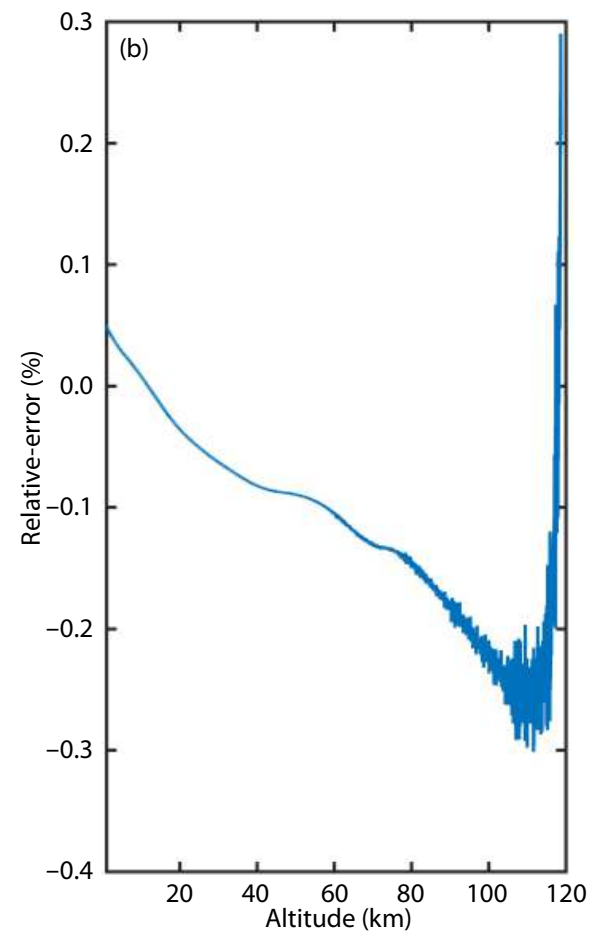

Figure 4. Variation in inversion results and relative error with altitude. (a) Molecular oxygen density decreases with altitude. (b) Relative error increases up to approximately $100 \mathrm{~km}$, and our value for the oxygen molecular number density becomes increasingly lower than the value determined by the MSISE00 model. 

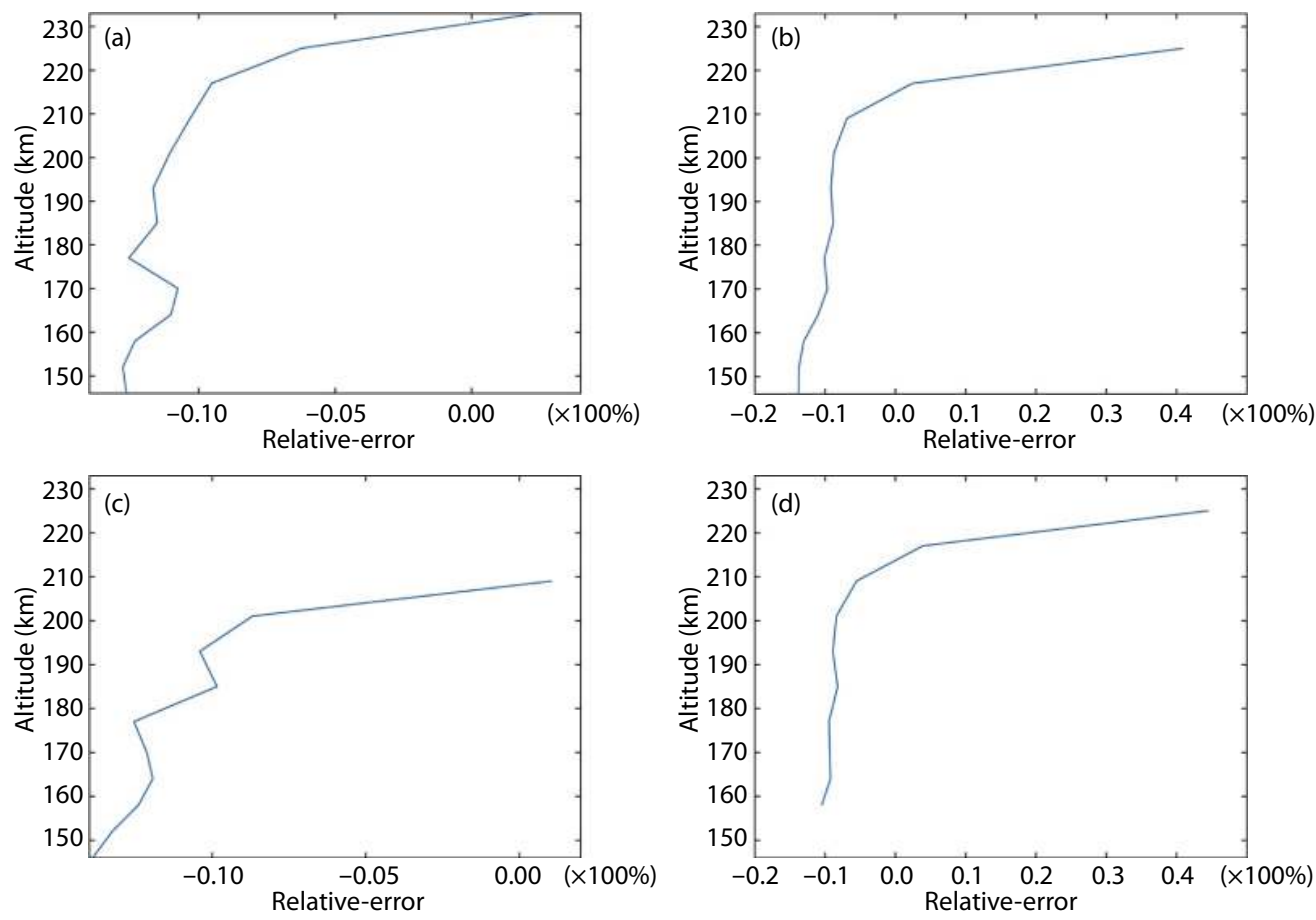

Figure 5. Variation in the relative error of our inversion results and the Level 2 results of GOLD for (a) March, (b) June, (c) September, and (d) December.

of the GOLD data. This deviation could be due to the $10 \%-20 \%$ error in the data released by GOLD (Siegmund et al., 2016) or the fact that the inversion method adopted by GOLD used the traditional solar occultation inversion algorithm. It is also possible that the value of the absorption cross-sectional area was sufficient to ignore the change in absorption cross section with altitude (Herman and Mentall, 1982). Finally, the deviation could be partly due to noise in the onion-peeling method. Overall, we consider the deviation of the inversion result to be within a reasonable range and that this result demonstrates the accuracy of our inversion algorithm.

\section{Effect of a Priori Data on Inversion Error}

We analyzed how the inversion error was affected by changes in the a priori data, including those for the temperature and oxygen density at the corresponding altitude from the MSISE00 atmospheric model. These atmospheric parameters are related to the season, latitude and longitude, and solar activity. Therefore, the error was calculated for different seasons, latitudes, and solar activities by using normal inversion; this was not compared with the MSISE00 results.

\subsection{Variation in Error Attributable to Season}

The monthly average input data for March, June, September, and December were used to calculate error (Figure 6).

Synthesis of the inversion errors showed that during all 4 months, the relative error gradually increased up to $37 \mathrm{~km}$ and was relatively flat (approximately 0-0.004\%) between 50 and $120 \mathrm{~km}$. In addition, the analysis showed that the inversion error was lowest in September (Figure 6c), with a peak error of $-0.001 \%$. This was because the start time of the occultation event was set to be
September (Sun MC et al., 2020a). However, the relative error at the same altitude for the 4 months was not significantly different, varying by at most $0.001 \%$. This result indicates that the inversion accuracy was not sensitive to the time of year.

\subsection{Variation in Error with Latitude}

The distribution characteristics of the occultation event showed very little change with longitude. Therefore, the latitude was varied while the other variables were kept constant. Figure 7 shows the inversion error at the equator, $45^{\circ}$, and $90^{\circ}$.

Figure 7 shows that the maximum inversion error values at $0^{\circ}, 45^{\circ}$, and $90^{\circ}$ were $0.15 \%, 0.08 \%$, and $0.09 \%$, respectively. The largest inversion error was observed at the equator because at this latitude, the oxygen content is significantly higher than that at other latitudes; therefore, the oxygen number density obtained by the inversion was closer to the actual atmospheric value. Because the original input data remained constant, the denominator of the relative error increased, which resulted in an increase in error. No clear difference was observed in the error values at $45^{\circ}$ and $90^{\circ}$, suggesting no specific trend in the change in error with latitude, which may be related to the latitude area covered by the occultation event. However, the variation in error between the equator and higher latitudes was several orders of magnitude greater than the variation in error attributable to seasonal variation. Therefore, the impact of latitude changes should be considered during the inversion process.

\subsection{Variation in Error with Solar Activity}

The $F_{10.7}$ index was used to represent solar activity because it can be used to distinguish between years of high and low solar activity. The annual trend in the $F_{10.7}$ index, based on an analysis of the 

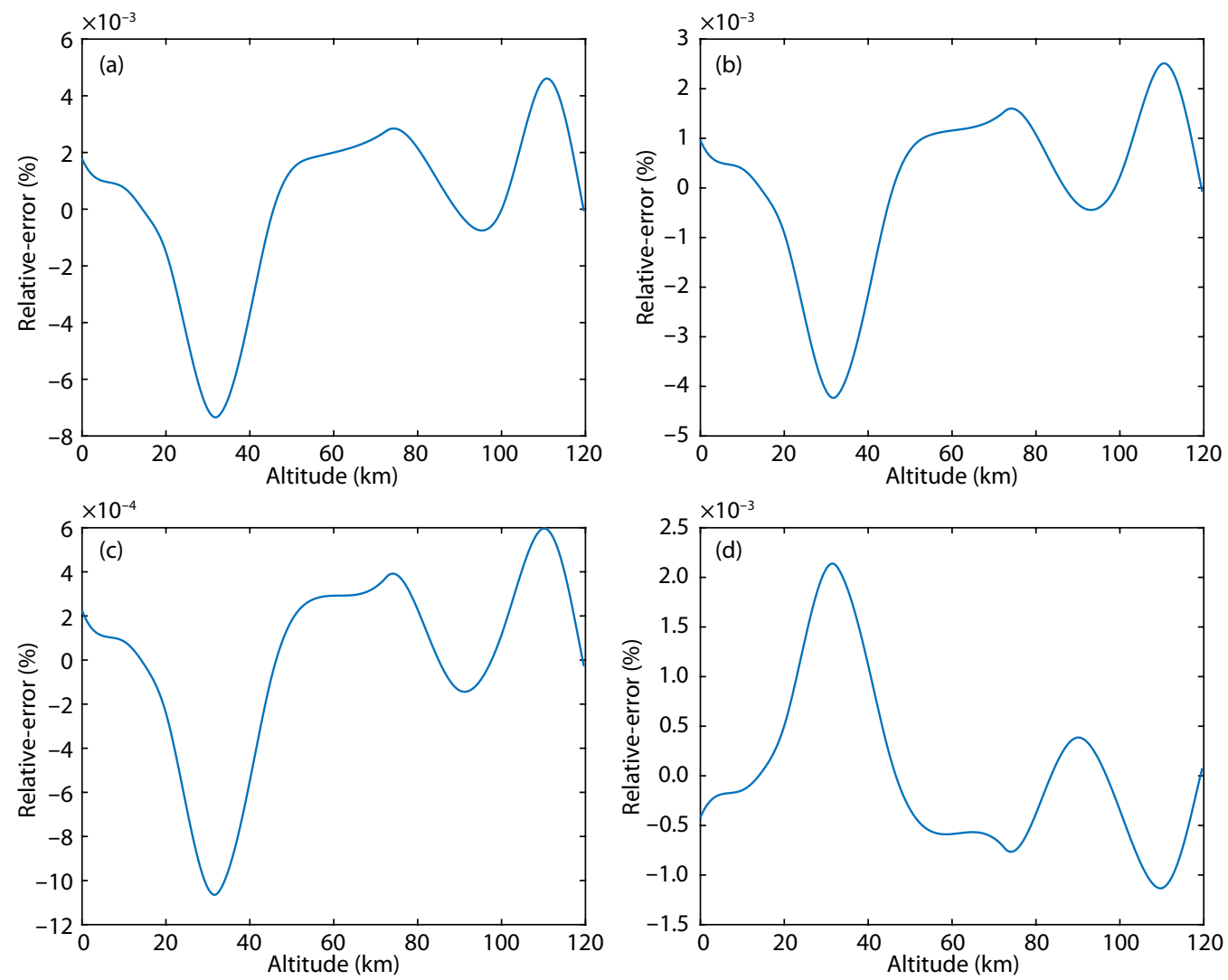

Figure 6. Relative error distribution with altitude in (a) March, (b) June, (c) September, and (d) December. The $y$-axis scale in panels (a), (b), and (d) are $\times 10^{-3}$, whereas the $y$-axis scale in panel (c) is $\times 10^{-4}$.
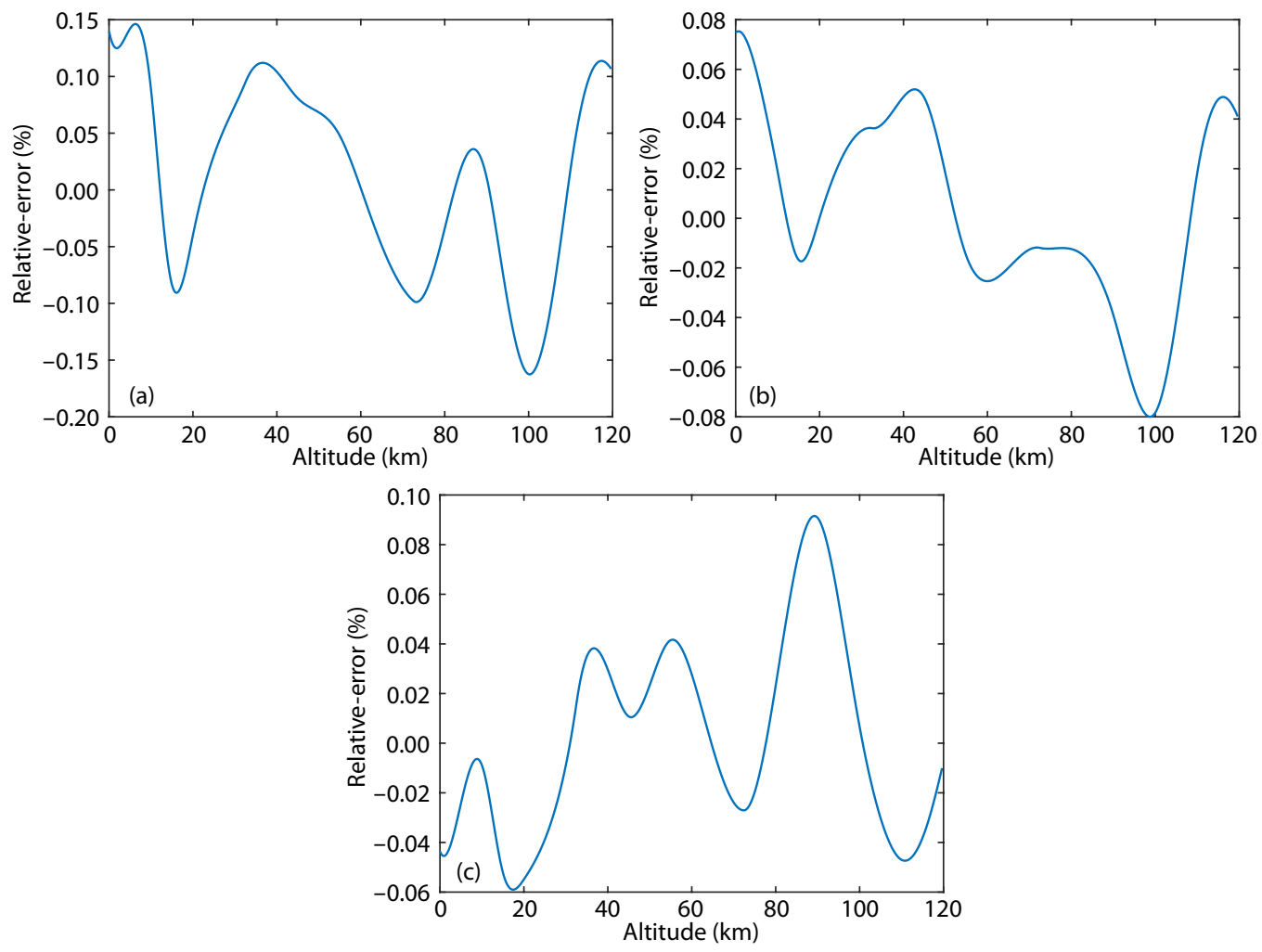

Figure 7. Variation in inversion error with latitude. (a) Equator, (b) $45^{\circ}$, and (c) $90^{\circ}$. 
Penticton observation data, is shown in Figure 8. The 2012-2014 period was one of high solar activity, in which solar fluxes reached 120-150 solar flux units (SFU), whereas 2009-2010 and 2017-2020 were periods of low solar activity, with highs of only approximately 70-90 SFU.

To analyze the effect of changes in solar activity on the inversion error, the relative error was calculated by using $F_{10.7}$ indexes of 70 and 150 SFU (Figure 9).

When solar activity was low (Figure 9a), the relative error was $0 \%$ up to an altitude of $60 \mathrm{~km}$, and it gradually increased to a maximum value of $0.05 \%$ between 60 and $120 \mathrm{~km}$. During high solar activity (Figure 9b), the relative error was $0 \%$ up to $40 \mathrm{~km}$, then it increased slightly and peaked at $0.01 \%$ at approximately $60 \mathrm{~km}$. The relative error between 60 and $100 \mathrm{~km}$ was the same as that when the solar activity was low; however, above $100 \mathrm{~km}$, the error during high solar activity was marginally lower than that during low solar activity. The difference in error during different solar activities occurred because during years of high solar activity, the oxygen-nitrogen ratio and the electron content increase and the oxygen molecule content decreases. This ultimately leads to a decrease in the density obtained from the inversion while the original oxygen molecule density remains unchanged. This results in an enlarged error at lower altitudes.

\section{Error Analysis of the Spectral Absorption Line Type}

In the inversion, the molecular absorption cross section was calcu- lated by line-by-line integration, which involved the absorption line type of the molecule. The absorption line type used in section 2 between 0 and $120 \mathrm{~km}$ was a Doppler line type. However, in the actual atmosphere, the main absorption lines are different at different altitudes. At altitudes below $20 \mathrm{~km}$, the spectral line broadens because of the broadening pressure, and the absorption line type at this altitude is the Lorentz line type (Equation (4)):

$$
\begin{gathered}
f_{L}\left(v-v_{0}\right)=\frac{1}{\pi} \frac{a_{L}}{\left(v-v_{0}\right)^{2}+a_{L}^{2}}, \\
a_{L}=0.1 \times \frac{p}{p_{0}}\left(\frac{T_{0}}{T}\right)^{0.5},
\end{gathered}
$$

where $a_{L}$ is the half-width of the spectral line, which is related to the temperature and pressure. At altitudes above $50 \mathrm{~km}$, the linear broadening mechanism is Doppler broadening, and only the Doppler linear pattern is considered in the calculation process (Equation (5)):

$$
\begin{gathered}
f_{D}\left(v-v_{0}\right)=\frac{1}{a_{D} \sqrt{\pi}} \exp \left(\left(\frac{v-v_{0}}{a_{D}}\right)^{2}\right), \\
a_{D}=v_{0}\left(\frac{2 k T}{m c^{2}}\right)^{\frac{1}{2}},
\end{gathered}
$$

where $m$ is the molecular mass and $k$ is the Boltzmann constant. Between 20 and $50 \mathrm{~km}$, the broadening of the spectral line is caused by a combination of the two effects. The Voigt profile was used to combine both line types to obtain the following line type

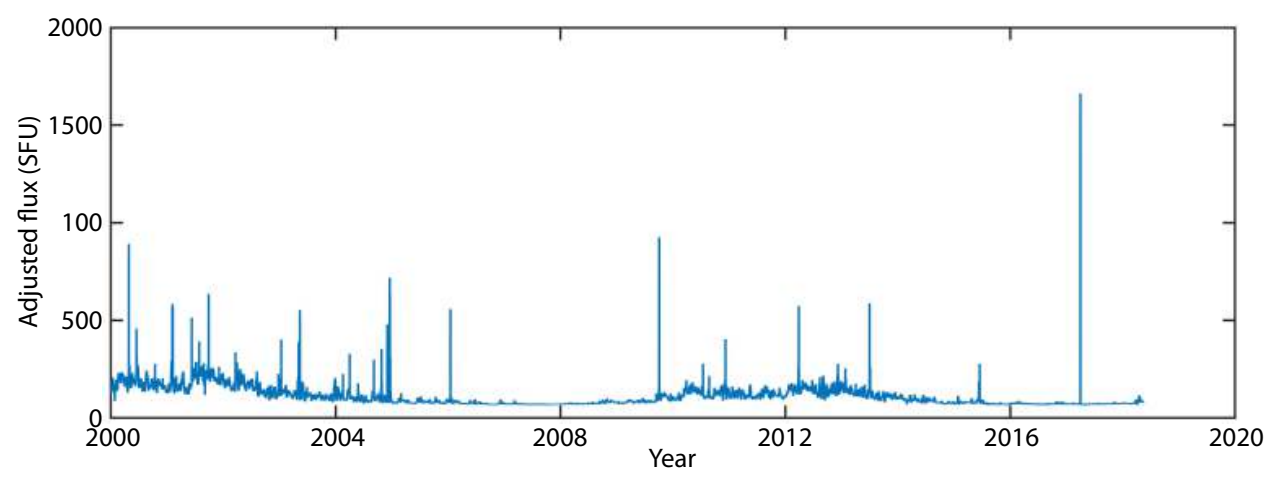

Figure 8. Annual change in the $F_{10.7}$ index from 2000 to 2020.
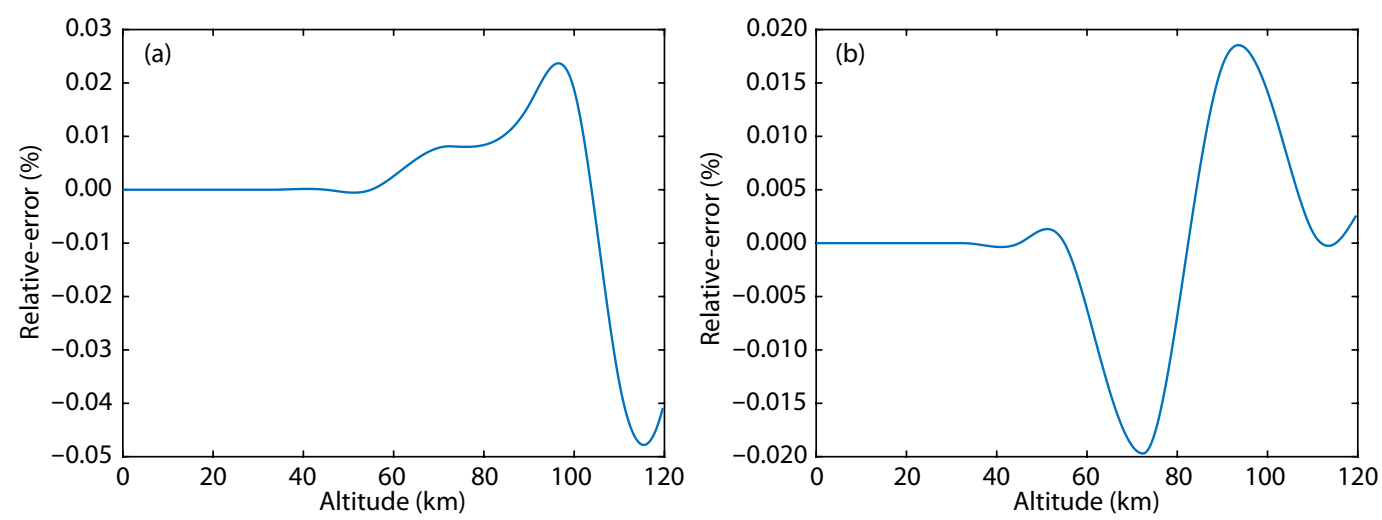

Figure 9. Variation in relative error with altitude under different solar activities. (a) 70 SFU; (b) 150 SFU. 
form (Equation (6)):

$$
\begin{gathered}
f_{v}\left(v-v_{0}\right)=\frac{1}{a_{D} \sqrt{\pi}} K(x, y), \\
K(x, y)=\frac{y}{\pi} \int_{-\infty}^{\infty} \frac{1}{y^{2}+(x-t)^{2}} \exp \left(-t^{2}\right) \mathrm{d} t
\end{gathered}
$$

where $y=\frac{a}{a_{D}}, x=\frac{v-v_{0}}{a_{D}}$, and $t=\frac{v-v^{\prime}}{a_{D}}$. Equation (7) was used to meet the normalization condition:

$$
\int_{-\infty}^{\infty} f_{v}\left(v-v_{0}\right) d\left(v-v_{0}\right)=1
$$

The inversion results of the corrected absorption cross section were compared with the uncorrected results to calculate the relative error (Figure 10).

It can be seen from Figure 10 that modification of the line type affects only the error below $50 \mathrm{~km}$, and this can result in an error of up to $1 \%$. Thus, it has a significantly greater impact on error than do the parameters discussed in Section 3; it is therefore the main parameter that can modify the error.

\section{Influence of the Earth's Ellipticity on the Error Distribution}

During the inversion process, we assumed that the Earth's local arcs were symmetrical. However, the Earth's actual atmosphere is elliptical, which will inevitably introduce error into the inversion results (Gong XY et al., 2007). Because this elliptical effect is more impactful at lower altitudes, we performed a local arc correction to eliminate the error. This was done by finding the ray where the altitude of the occultation event was closest to the Earth's surface. The center of the arc corresponding to the intersection of the plane and the ellipse of the Earth was then used as the center of

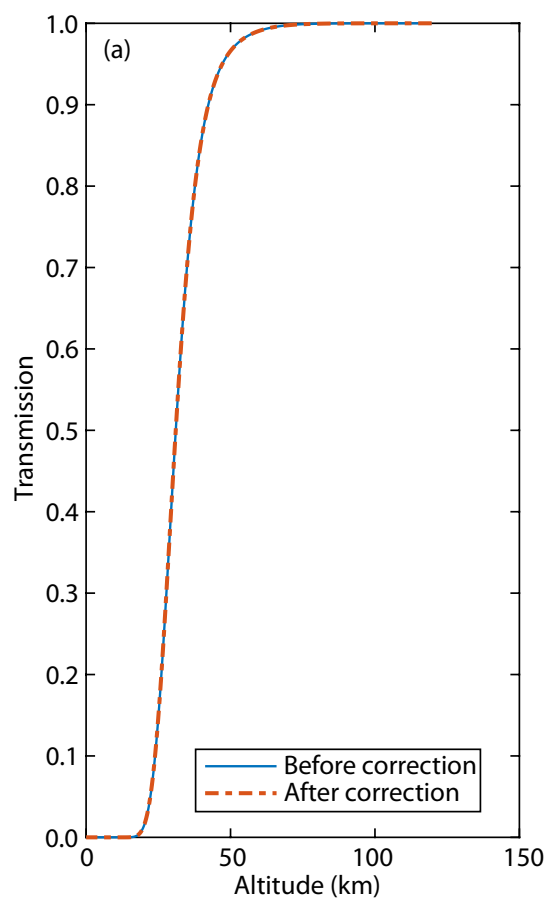

the local arc for the correction of an occultation event. The center of the arc varied for each occultation event as did the radius of the Earth. All the coordinate points, including the satellite positions and ray point positions, were translated to the coordinate system for the inversion. After calculation, the offsets of the arc center of the lowest altitude occultation event were $0.038752,0.006498$, and $42,831.165 \mathrm{~m}$, and the radius was $6,378,137 \mathrm{~m}$. Figure $11 \mathrm{a}$ shows the change in transmittance with altitude before and after correction. Figure $11 \mathrm{~b}$ shows the relative error between the results after and before correction for ellipticity. The corrected transmittance was used to perform the inversion, and the error was calculated relative to the inversion results by using the uncorrected transmittance (Figure 12).

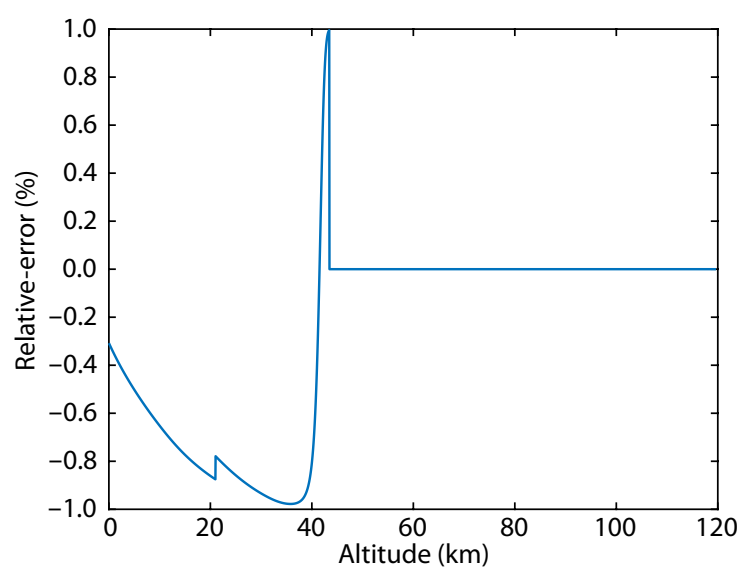

Figure 10. Change in relative error with altitude after absorption line correction. The error varies from -1 to $1 \%$ below $50 \mathrm{~km}$ but is $0 \%$ above $50 \mathrm{~km}$.

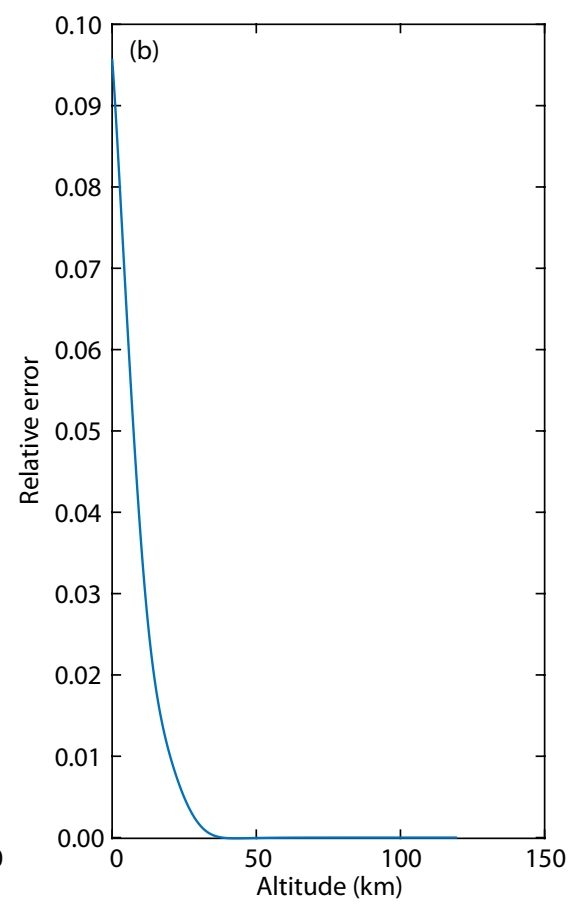

Figure 11. (a) Transmittance before and after correction for ellipticity. (b) Error relative to the MSISEO0 model when using the transmittance corrected for ellipticity in the inversion. 


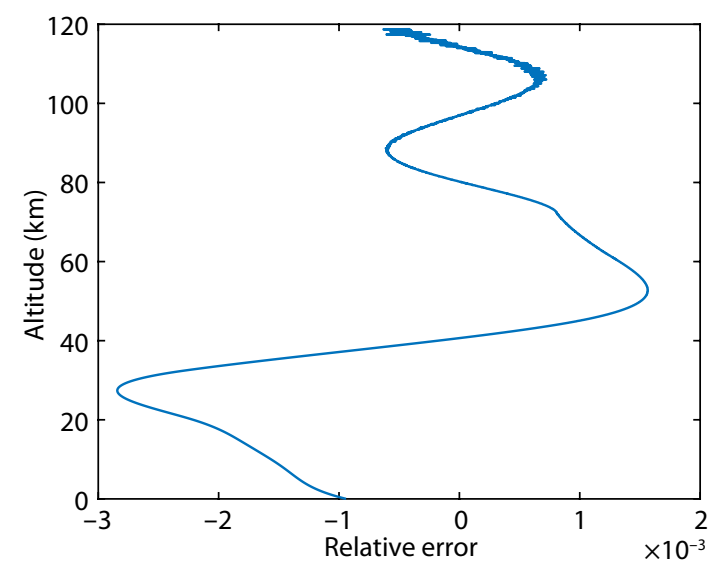

Figure 12. Relative error of the elliptically corrected inversion results compared with the uncorrected inversion results.

Figure 11 shows that the error in the simulated transmittance before and after the correction decreases with altitude and that the error is greatest at the Earth's surface, with a value of $0.1 \%$. This is because as the altitude increases, the curvature effect of the Earth gradually decreases, and at higher altitudes, this effect can be ignored. Figure 12 shows that the arc correction does not result in significant decreases in error, as the maximum error is less than $0.003 \%$. Therefore, the influence of the Earth's ellipticity can be ignored during the inversion process.

\section{Conclusions}

In this study, a stellar occultation inversion algorithm for a single wavelength of light $(761.4 \mathrm{~nm})$ was constructed based on the onion-peeling method. The simulation data were inverted to obtain the oxygen number density, which was then compared with the results from the MSISEO0 atmospheric model. This comparison was used to calculate the inversion accuracy. The results showed that the error is within a reasonable range, which verifies the practicability of our inversion algorithm. The average monthly relative deviation was also analyzed to further verify the algorithm.

The effect of variation in the type of absorption line and the influence of the Earth's ellipticity and a priori data (season, latitude, and solar activity) on the accuracy of the inversion model were also analyzed. Variation in the type of absorption line had the greatest impact on the inversion results, with error values of up to $1 \%$. The Earth's ellipticity had a measurable impact on the simulated transmittance; however, it had little effect on the inversion results and resulted in only $0.003 \%$ error. The influence of seasons on the error was on the order of $0.001 \%$; however, the influences of latitude and solar activity were more significant, producing maximum errors of $0.15 \%$ and $0.05 \%$, respectively. Thus, we believe that it is important consider the type of absorption line, latitude, and solar activity during the inversion process. By comparison, the need to consider ellipticity and season is not as critical.

The onion-peeling method presented here contained measurable noise and is suitable for only single-wavelength inversion. Moreover, inversion wavelength selection, satellite orbits, instrument effects, and stray light are factors that will affect the accur- acy of the inversion, but they were not considered in our model. It is our intention to address these shortcomings and factors in future studies.

\section{Acknowledgments}

We would like to thank Hu Xiong, National Center for Space Science, Chinese Academy of Sciences, for his assistance and guidance. We also thank GOLD and Penticton for the observation data provided. This study was supported in part by the National Natural Science Foundation of China (NSFC) under Grant 61971385.

\section{References}

Bertaux, J. L., Hauchecorne, A., Dalaudier, F., Cot, C., Kyrölä, E., Fussen, D., Tamminen, J., Leppelmeier, G. W., Sofieva, V., ... Fraisse, R. (2004). First results on GOMOS/ENVISAT. Adv. Space Res., 33(7), 1029-1035. https://doi.org/10.1016/j.asr.2003.09.037

Bertaux, J. L., Vandaele, A. C., Korablev, O., Villard, E., Fedorova, A., Fussen, D., Quemerais, E., Belyaev, D., Mahieux, A., ... the SPICAV/SOIR team. (2007). A warm layer in Venus' cryosphere and high-altitude measurements of $\mathrm{HF}$, $\mathrm{HCl}, \mathrm{H}_{2} \mathrm{O}$ and HDO. Nature, 450(7170), 646-649.

https://doi.org/10.1038/nature05974

Bertaux, J. L., Kyrölä, E., Fussen, D., Hauchecorne, A., Dalaudier, F., Sofieva, V., Tamminen, J., Vanhellemont, F., d'Andon, O. F., ... Fraisse, R. (2010). Global ozone monitoring by occultation of stars: an overview of GOMOS measurements on ENVISAT. Atmos. Chem. Phys., 10(24), 12091-12148. https://doi.org/10.5194/acp-10-12091-2010

DeMajistre, R., and Yee, J. H. (2002). Atmospheric remote sensing using a combined extinctive and refractive stellar occultation technique 2 . Inversion method for extinction measurements. J. Geophys. Res. : Atmos., 107(D15), ACH 6-1-ACH 6-18. https://doi.org/10.1029/2001JD000795

Eastes, R. (2009). NASA mission to explore forcing of Earth's space environment. Eos Trans. AGU, 90(18), 155. https://doi.org/10.1029/2009EO180002

Eastes, R. W., McClintock, W. E., Burns, A. G., Anderson, D. N., Andersson, L., Codrescu, M., Correira, J. T., Daniell, R. E., England, S. L., ... Oberheide, J. (2017). The global-scale observations of the limb and disk (GOLD) mission. Space Sci. Rev., 212(1), 383-408. https://doi.org/10.1007/s11214-017-0392-2

Festou, M. C., Atreya, S. K., Donahue, T. M., Sandel, B. R., Shemansky, D. E., and Broadfoot, A. L. (1981). Composition and thermal profiles of the Jovian upper atmosphere determined by the Voyager Ultraviolet Stellar Occultation Experiment. J. Geophys. Res. :Space Phys., 86(A7), 5715-5725. https://doi.org/10.1029/JA086iA07p05715

Forget, F., Montmessin, F., Bertaux, J. L., González-Galindo, F., Lebonnois, S., Quémerais, E., Reberac, A., Dimarellis, E., and López-Valverde, M. A. (2009). Density and temperatures of the upper Martian atmosphere measured by stellar occultations with Mars Express SPICAM. J. Geophys. Res. :Planets, 114(E1), E01004. https://doi.org/10.1029/2008JE003086

Gong, X. Y., Hu, X., Wu, X. C., and Zhang, X. X. (2007). Preliminary analysis of error characteristics in atmospheric inversion of GPS radio occultation. Chin. J. Geophys., 50(4), 1017-1029. https://doi.org/10.3321/j.issn:00015733.2007.04.009

Greer, K. R., England, S. L., Becker, E., Rusch, D., and Eastes, R. (2018). Modeled gravity wave-like perturbations in the brightness of far ultraviolet emissions for the GOLD mission. J. Geophys. Res. :Space Phys., 123(7), 5821-5830. https://doi.org/10.1029/2018JA025501

Hays, R. G., and Roble, P. B. (1968). Stellar spectra and atmospheric composition. J. Atomos. Sci., 25, 1141-1153.

Herman, J. R., and Mentall, J. E. (1982). O2 absorption cross sections (187-225 $\mathrm{nm}$ ) from stratospheric solar flux measurements. J. Geophys. Res. :Oceans, 87(C11), 8967-8975. https://doi.org/10.1029/JC087iC11p08967

Kyrölä, E., Tamminen, J., Leppelmeier, G. W., Sofieva, V., Hassinen, S., Bertaux, J. L., Hauchecorne, A., Dalaudier, F., Cot, C., ... Vanhellemont, F. (2004). GOMOS on Envisat: an overview. Adv. Space Res., 33(7), 1020-1028. https://doi.org/10.1016/S0273-1177(03)00590-8

Kyrölä, E., Tamminen, J., Sofieva, V., Bertaux, J. L., Hauchecorne, A., Dalaudier, F., 
Fussen, D., Vanhellemont, F., D'Andon, O. F., ... Fraisse, R. (2010). Retrieval of atmospheric parameters from GOMOS data. Atmos. Chem. Phys., 10(23), 11881-11903. https://doi.org/10.5194/acp-10-11881-2010

Lumpe, J. D., Floyd, L. E., Herring, L. C., Gibson, S. T., and Lewis, B. R. (2007). Measurements of thermospheric molecular oxygen from the Solar Ultraviolet Spectral Irradiance Monitor. J. Geophys. Res. :Atmos., 112(D16), D16308. https://doi.org/10.1029/2006JD008076

Lumpe, J. D. , Correira, J. , Evans, J. S. , Eastes, R. , McClintock, B. , and Beland, S. (2016). Measurements of thermospheric $\mathrm{O}_{2}$ density from GOLD. In AGU Fall Meeting. American Geophysical Union.

Ning, L. X., Wang, H. M., Cheng, P., Chu, Y. N., and Cao, D. Z. (2001). Research development on kinetic behavior of the Herzberg states of $\mathrm{O}_{2}$ in the upper atmosphere. Chin. J. Quant. Electr., 18(1), 9-15. https://doi.org/10.3969/j.issn.1007-5461.2001.01.002

Qi, R. B., He, S. K., Li, X. T., and Wang, X. Z. (2015). Simulation of TDLAS direct absorption based on HITRAN database. Spectrosc. Spect. Anal., 35(1), 172-177. https://doi.org/10.3964/j.issn.1000-0593(2015)01-0172-06

Qian, L. Y., Solomon, S. C., and Kane, T. J. (2009). Seasonal variation of thermospheric density and composition. J. Geophys. Res. :Space Phys., 114(A1), A01312. https://doi.org/10.1029/2008JA013643

Ratier, G., Levrini, G., Popescu, A., Paulsen, T., Readings, C., and Langen, J. (1999). GOMOS: Envisat's contribution to trace gas measurements. Air Space Eur., 1(5-6), 33-39. https://doi.org/10.1016/S1290-0958(00)88868-1

Roble, R. G., and Hays, P. B. (1972). A technique for recovering the vertical number density profile of atmospheric gases from planetary occultation data. Planet. Space Sci., 20(10), 1727-1744. https://doi.org/10.1016/00320633(72)90194-8

Siegmund, O. H. W., McPhate, J., Curtis, T., Jelinsky, S., Vallerga, J. V., Hull, J., and Tedesco, J. (2016). Ultraviolet imaging detectors for the GOLD mission. Proc. SPIE, 9905, 99050D. https://doi.org/10.1117/12.2232219

Sun, M. C., Wu, X. C., and Hu, X. (2020a). Analysis of simulation results of orbit observation of stellar occultation technology. Spectrosc. Spect. Anal., 40(1), 298-304. https://doi.org/10.3964/j.issn.1000-0593(2020)01-0298-07

Sun, M. C., Wu, X. C., Gong, X. Y., and Hu, X. (2020b). Transmittance simulation calculation based on $3 \mathrm{D}$ ray tracing and HITRAN database. Spectrosc. Spect. Anal., 40(7), 2092-2097. https://doi.org/10.3964/j.issn.1000-0593(2020)072092-06

Swartz, W. H., Yee, J. H., Vervack, R. J. Jr., Lloyd, S. A., and Newman, P. A. (2002). Photochemical ozone loss in the Arctic as determined by MSX/UVISI stellar occultation observations during the 1999/2000 winter. J. Geophys. Res.:Atmos., 107(D20), 8296. https://doi.org/10.1029/2001JD000933

Yee, J. H., Vervack, R. J. Jr., DeMajistre, R., Morgan, F., Carbary, J. F., Romick, G. J., Morrison, D., Lloyd, S. A., DeCola, P. L., ... Meng, C. I. (2002). Atmospheric remote sensing using a combined extinctive and refractive stellar occultation technique 1. Overview and proof-of-concept observations. J. Geophys. Res., 107(D14), 4213. https://doi.org/10.1029/2001JD000794

Zhang, H., and Shi, G. Y. (2000). A fast and efficient line-by-line calculation method for atmospheric absorption. Chin. J. Atmos. Sci., 24(1), 111-121. https://doi.org/10.3878/j.issn.1006-9895.2000.01.12 